\title{
Study on the Value of DCE-MRI in Differentiating Glossitis and Tongue Cancer and the Intratumour Heterogeneity
}

\section{Fenghai Liu \\ Meng Zhao \\ Shan Lu \\ Liqing Kang $\mathbb{D}$}

Department of Magnetic Resonance Imaging, Cangzhou Central Hospital, Cangzhou, Hebei Province, 06100I,

People's Republic of China
Correspondence: Liqing Kang Department of Magnetic Resonance Imaging, Cangzhou Central Hospital, No. 16 of Xinhua West Road, Cangzhou, 06100I, Hebei Province, People's

Republic of China

Tel +86 I3333367921

Email 13333367921@I63.com
Objective: DCE-MRI is an imaging technique that reflects the blood perfusion status of the tissue's microcirculation. The purpose of this article is to explore the clinical value of dynamic contrast-enhanced (DCE)-MRI in distinguishing benign and malignant tongue lesions and the internal heterogeneity of a tumour.

Methods: The patients were divided into a tongue cancer group (22 patients) and a glossitis group ( 7 patients) based on the pathology results. All of the patients underwent DCE-MRI examination.

Results: The results of this study showed that the volume transfer constant (Ktrans), rate constant (Kep), contrast enhancement ratio (CER) and initial area under the gadolinium contrast agent concentration time curve (IAUGG) values of the tongue cancer group were significantly higher than those of the glossitis group, and the difference was statistically significant $(\mathrm{P}<0.05)$. However, the extravascular extracellular volume fraction $(\mathrm{Ve})$, fractional plasma volume (fPV), maximum slope (MaxSlope), and bolus arrival time (BAT) values measured by DCE-MRI in the tongue cancer group were not significantly different from those in the glossitis group $(\mathrm{P}>0.05)$. The results of this study showed that the Ktrans, Kep, and IAUGG values measured by DCE-MRI had a good ability to distinguish tongue inflammation from tumours and Ktrans threshold of 0.484 has the best discriminative ability among them. The mean Ktrans values of stage I-II lesions were significantly higher than that of stage III-IV lesion $(\mathrm{p}=0.045)$.

Conclusion: DCE-MRI is effective in distinguishing between benign and malignant tongue lesions and the internal heterogeneity of the tumour; it is worth following up in a larger study.

Clinical Registration Number: Research registry 6393.

Keywords: DCE-MRI, tongue cancer, tumour heterogeneity, tumour microenvironment, tumour precision diagnosis and treatment

\section{Introduction}

Tongue cancer (TC) is a common cancer among head and neck cancers (HNC), ${ }^{1-4}$ its morbidity and mortality being ranked first in oral cavity cancers (OCC), accounting for $25-40 \%$ of oral malignancies, 5,6 and it is showing a trend of occurring in younger patients. ${ }^{7}$ Long-term chronic irritation, smoking, drinking, betel nut, human papillomavirus (HPV) infection, etc. are the main risk factors. ${ }^{8-10}$ TC mostly occurs in the first two-thirds of the tongue. It mainly consists of squamous cell carcinoma (SCC), with a high degree of malignancy. The incidence 
of recurrence and metastasis is high, and the prognosis is poor. Due to the unique tissue structure of the tongue (mainly composed of muscles and rich in lymphatic vessels), TC is more aggressive than other oral tumours and is more prone to lymph node metastasis. Therefore, the use of non-invasive imaging methods for comprehensive evaluation of tumour types and differentiation of levels, internal heterogeneity and microenvironmental characteristics before surgery is of great significance for clinical decisionmaking, patient management, and prognostic judgment.

In a wider sense, glossitis can mean inflammation of the tongue generally. ${ }^{11}$ Glossitis is often caused by nutritional deficiencies and may be painless or cause discomfort. Classification of glossitis include atrophic glossitis, median rhomboid glossitis, benign migratory glossitis, geometric glossitis and strawberry tongue. ${ }^{12}$ The diagnosis of glossitis is mainly based on clinical manifestations, and the etiology should be sought as far as possible. Glossitis is self-limited or can be cured by treatment of the cause, whereas TC cannot. When necessary, biopsy is taken to rule out malignancy. ${ }^{13}$ Previous histopathological examination is the gold standard for evaluating tumour heterogeneity, but the information with regard to tumour tissue and microenvironment obtained by puncture biopsy or surgery is greatly limited, being unable to obtain the overall situation of the tumour tissue, having poor repeatability, being connected with certain traumas and risks, and being subject to invasive examination. However, the role of traditional imaging techniques (including Computer Tomography (CT) and conventional static Magnetic Resonance Imaging (MRI)) in differentiating benign and malignant tumours is controversial, neither can they quantify tumour heterogeneity, nor can they reflect the changes in the internal and surrounding microenvironment of the tumour. $^{14-16}$ Dynamic contrast-enhanced (DCE)-MRI is an imaging technology that reflects the blood perfusion status of the tissue's microcirculation. After analysis and calculation, a series of semiquantitative and quantitative parameters can be obtained, including volume transfer constant (Ktrans), extravascular extracellular volume fraction (Ve), rate constant (Kep), maximum slope (MaxSlope), fractional plasma volume (fPV), contrast enhancement ratio (CER), initial area under the gadolinium contrast agent concentration time curve (IAUGG), bolus arrival time (BAT) and so on; then, microcirculation characteristics such as blood volume, blood flow, and microvascular permeability in the tumour tissue can be quantitatively analysed. ${ }^{17}$
At present, several studies have reported the value of DCE-MRI in HNC. ${ }^{18,19}$ While among HNC, TC has relatively early metastasis, a high recurrence rate, and a poor prognosis. Therefore, this study intends to explore the clinical value of DCE-MRI in distinguishing benign and malignant tongue lesions and the internal heterogeneity of the tumour, and provide a theoretical basis for the early clinical diagnosis of TC.

\section{Methods}

\section{Research Object}

In this study, the main research objects were patients with suspected glossitis or TC admitted to our hospital. Clinical diagnosis was made according to clinical manifestations (symptoms, lesion location, lesion characteristics), etiology, oral examination, laboratory examination, etc. When the diagnosis was difficult, a biopsy was taken to confirm the diagnosis. The patients were divided into a TC group and a glossitis group based on the clinical diagnosis and postoperative pathology results. All patients underwent DCE-MRI examination and the clinical value of DCE-MRI in distinguishing benign and malignant tongue lesions and the internal heterogeneity of the tumour in the two groups of patients was observed. This study complies with the Declaration of Helsinki of the World Medical Association and has been approved by the ethics committee of Cangzhou Central hospital (IRB: 2017-027). All patients signed an informed consent form.

\section{Inclusion and Exclusion Criteria}

Inclusion criteria: (1) MRI examination within one week before surgery, including conventional sequence and DCEMRI sequence; (2) complete surgical and pathological examination results; (3) not receiving any anti-tumour treatment; (4) effective parameter values could be measured on the MRI image; (5) aged over 18 years; (6) patients having signed the informed consent.

Exclusion criteria: (1) intraoral dentures and other metal influences that caused severe image signal loss; (2) those who had contraindications to MRI examinations or those who were allergic to contrast agents and had not undergone an MRI enhancement scan; (3) patients with the DCE sequence missing or unable to complete the examination due to the patient's inability to cooperate; (4) patients with incomplete case data. 


\section{MR Scanning Equipment and Conventional Scanning Sequence}

All patients underwent oral MRI scan and DCE-MRI. The American GE 750W 3.0T MR scanner and the head and neck joint coil were used, and the patient was placed in a supine position. The MR routine scanning sequence included: FSE-T1WI axis position $(\mathrm{TR}=488 \mathrm{~ms}, \mathrm{TE}=$ Min Full, slice thickness $=4.0 \mathrm{~mm}$, slice interval $=$ $1.0 \mathrm{~mm}, \mathrm{FOV}=22 \mathrm{~cm} \times 22 \mathrm{~cm}$, flip angle $=142$, echo chain $(E T L)=3$, matrix $=228 \times 224$, NEX $=2$, bandwidth $=35.7)$; Ideal-T2WI-axis position $(\mathrm{TR}=3360 \mathrm{~ms}$, $\mathrm{TE}=$ $68 \mathrm{~ms}$, layer thickness $=4 \mathrm{~mm}$, layer interval $=1.0 \mathrm{~mm}$, $\mathrm{FOV}=22 \times 22 \mathrm{~cm}$, flip angle $=142$, echo chain $(\mathrm{ETL})=$ 16 , matrix $320 \times 256, \mathrm{NEX}=2$, bandwidth $=62.5$ ); IdealT2WI- sagittal/coronal position $(\mathrm{TR}=2870 \mathrm{~ms}, \mathrm{TE}=68$ $\mathrm{ms}$, slice thickness $=4 \mathrm{~mm}$, slice interval $=1.0 \mathrm{~mm}, \mathrm{FOV}=$ $22 \times 22 \mathrm{~cm}$, flip angle $=142$, echo chain $(\mathrm{ETL})=16$, matrix $288 \times 192$, NEX $=1$, bandwidth $=62.5$ ); STIR-DWI-axis position $(b=600, T R=2870 \mathrm{~ms}, \mathrm{TE}=$ Minimum, layer thickness $=4 \mathrm{~mm}$, layer interval $=1.0 \mathrm{~mm}$, FOV $=$ $22 \times 22 \mathrm{~cm}$, Inv Time $=249$, matrix $96 \times 96$, NEX $=2$, bandwidth $=250$ ); enhanced scan uses contrast agent GdDTPA $(0.1 \mathrm{mmol} / \mathrm{kg})$ through cubital vein injection and ideal-T1WI is performed for transverse, sagittal, and coronal scanning $(\mathrm{TR}=488 \mathrm{~ms}$, $\mathrm{TE}=$ Min Full, slice thickness $=4.0 \mathrm{~mm}$, slice interval $=1.0 \mathrm{~mm}, \mathrm{FOV}=22 \times 22 \mathrm{~cm}$, flip angle $=142$, echo chain $(E T L)=3$. Matrix $=288 \times$ 224 , NEX $=2$, bandwidth $=35.7$ ).

\section{DCE-MRI Scan Sequence and Parameters}

Using axial LAVA (three-dimensional volume interpolation fast spoiler gradient return sequence): TR $4.3=\mathrm{ms}$, $\mathrm{TE}=1.4 \mathrm{~ms}$, NEX 1, layer thickness $=5.0 \mathrm{~mm}$, matrix $160 \times 128$, FOV $22 \times 22 \mathrm{~cm}$, flip angle $=15$ degrees, bandwidth $=62.5$. 65 -period uninterrupted scanning under free breathing, single-phase scanning time is $5 \mathrm{~s}$, contrast medium is injected in the third period, Gd-DTPA $0.1 \mathrm{mmol} / \mathrm{kg}, 20 \mathrm{~mL}$ physiological saline is injected through the cubital vein at a flow rate of $3 \mathrm{~mL} / \mathrm{s}$, and the tube is flushed at the same rate.

All the DCE-MRI scan images were transferred to a GE AW 4.7 workstation in the United States, and special software was used for postprocessing. DCE-MRI quantitative parameter measurement: open the Gen IQ software to select the volume calculation range to include all tumours. Select tumour ROI and obtain the quantitative perfusion parameters: Ktrans, Ve, Kep, MaxSlope, fPV,
CER, IAUGG, BAT. The ROI selection refers to the MRI plain scan and enhancement, and the area with the largest parenchymal part and the most obvious enhancement on the map was selected to avoid artifacts, necrosis, and teeth. To reduce the human error caused by the measurement, each group of images was measured repeatedly three times and averaged. Finally, the postprocessing image was saved and each parameter value was recorded.

\section{Postoperative Pathological Examination}

Based on postoperative pathological results, only SCC was included in this study. The pathological TNM staging of SCC was recorded. TNM staging was conducted according to the 8th AJCC staging system. ${ }^{20}$ Due to the limitation of sample size, the TC group was divided into the early stage group (stage I-II) and the advanced stage group (stage III-IV).

\section{Statistical Methods}

In this study, SPSS 17.0 statistical software was used for data processing, and the measurement data were expressed as mean \pm standard deviation $(\bar{x} \pm s)$. The counting data were expressed as percentages (\%). The DCE-MRI parameter values of the glossitis and TC groups were compared in pairs. The independent-sample $t$-test was used for the data set that conformed to the normal distribution and had a uniform variance. Non-parametric testing was used for the data set that did not conform to the normal distribution or that had variance that was not uniform. The chi-square test was used for the counting data. The statistically significant perfusion parameters were screened out, and the receiver characteristic curve (ROC) was drawn to analyse the value of each parameter to determine the diagnostic efficacy of benign and malignant lesions, and to determine the corresponding diagnostic threshold (cut-off value). The sensitivity, specificity, positive predictive value, negative predictive value, and the cut-off value of each parameter were calculated for identifying benign and malignant lesions. Spearman rank correlation and binary logistic regression were used to analyse the correlation between the DCE-MRI parameters and the degree of differentiation of TC. $P<0.05$ indicated that the difference was statistically significant.

\section{Results}

\section{General Information}

The study finally included 29 patients with tongue lesions that met the conditions. According to the clinical diagnosis and postoperative pathology results, the patients were 


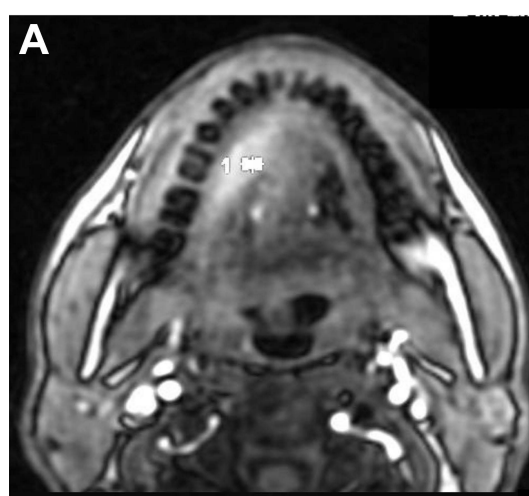

D

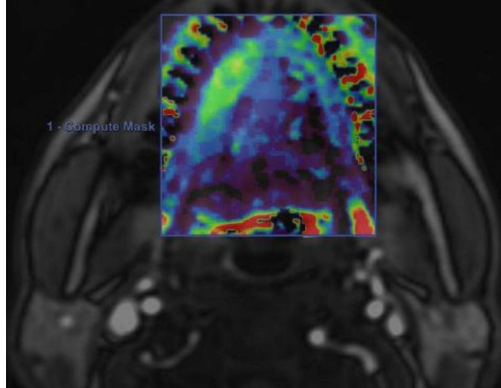

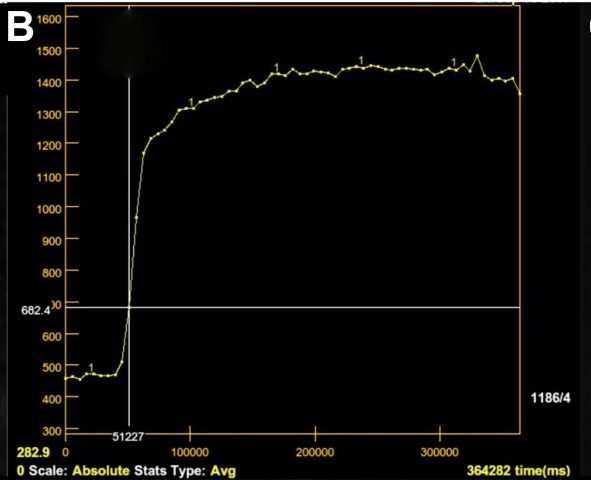

E

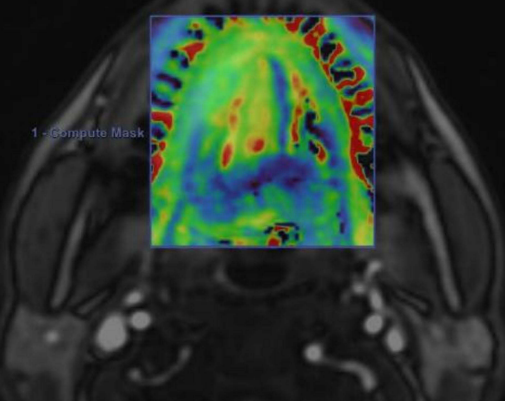

C

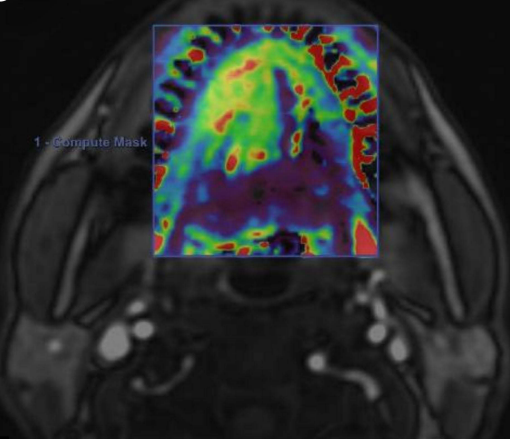

$\mathbf{F}$

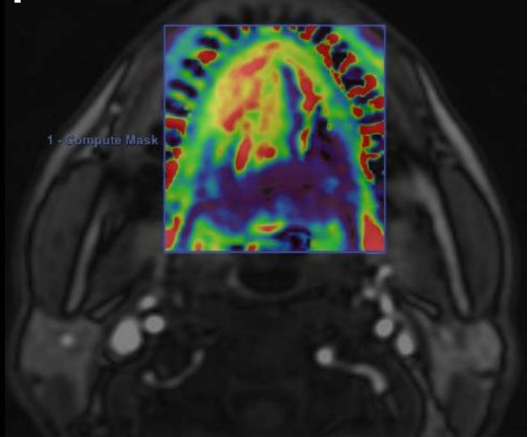

Figure I Picture of a patient with typical glossitis. Dynamic enhanced image (A), showing obvious enhancement in the lesion area and slight enhancement in the adjacent area; The lesion showed a plateau enhancement curve in ROI (B); Ktrans function map (C) owed that Ktrans value increased in the lesion area and adjacent area, especially in the lesion area. Ep functional map (D) showed significant increase only in the lesion area. The results of CER (E) and IAUGC (F) function maps were similar to those of Ktrans function maps, indicating that the corresponding perfusion parameters in the lesion area and adjacent areas were significantly increased.
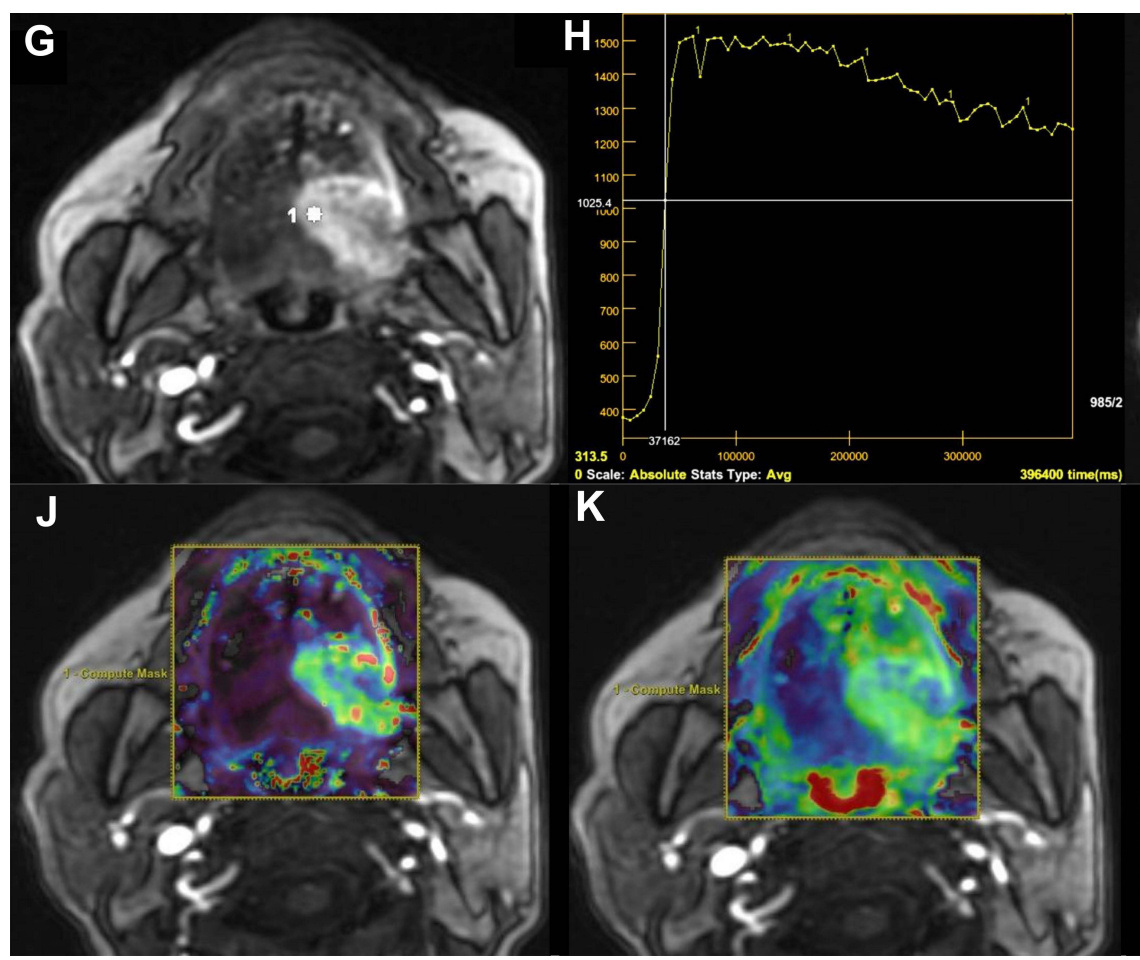

$\mathbf{K}$

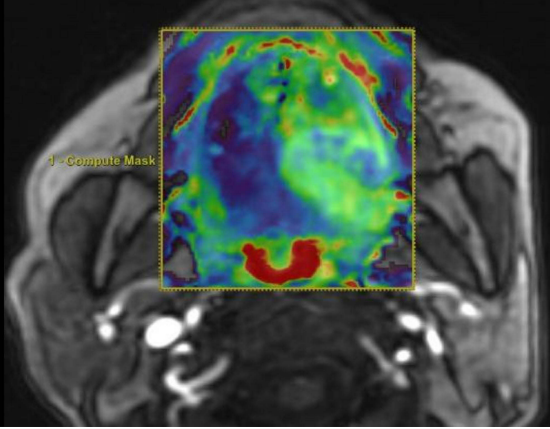

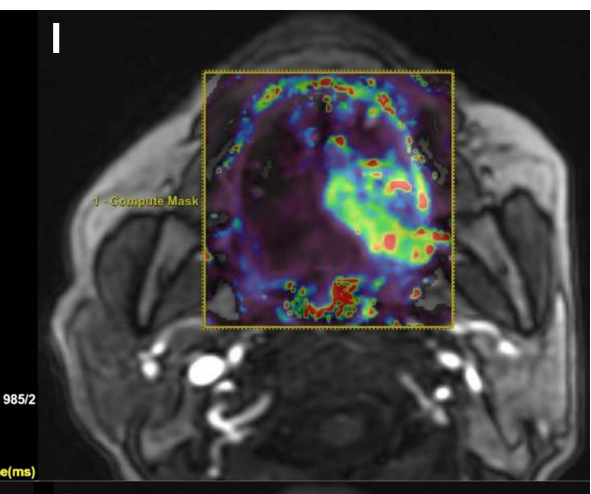

$\mathbf{L}$

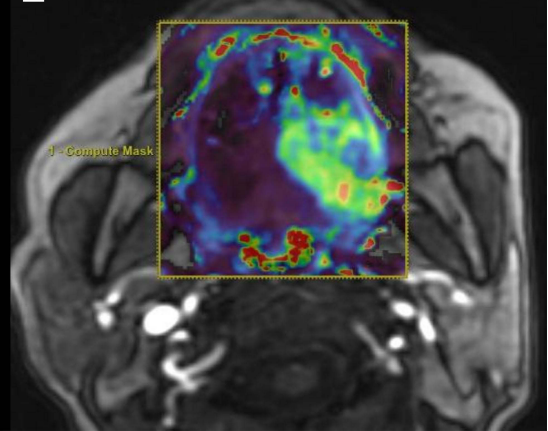

Figure 2 Picture of a typical tongue cancer patient. Dynamic enhanced image (G) showed obvious uneven enhancement with clear boundary. The selected dynamic enhancement curve in ROI presents a clearance type $(\mathbf{H})$; i I are the corresponding function maps of Ktrans, Kep, CER and IAUGC, respectively, showing that each perfusion parameter of the lesion was significantly higher than that of the adjacent tissues, and the distribution of each perfusion parameter in the lesion was uneven. 
Table I Clinical and Demographic Comparison Between the Two Groups

\begin{tabular}{|l|l|l|l|l|}
\hline Parameter & $\begin{array}{l}\text { Glossitis } \\
\text { Group } \\
(\mathbf{n = 7})\end{array}$ & $\begin{array}{l}\text { Tongue } \\
\text { Cancer Group } \\
(\mathbf{n = 2 2})\end{array}$ & $\mathbf{T} / \mathbf{X}^{2}$ & $P$ value \\
\hline $\begin{array}{c}\text { Gender } \\
\text { Male } \\
\text { Female }\end{array}$ & 2 & 16 & 0.004 & 0.947 \\
\hline Age & $53.71 \pm 16.19$ & $61.59 \pm 12.96$ & -1.321 & 0.198 \\
\hline BMI & $24.23 \pm 2.82$ & $22.91 \pm 3.45$ & 0.915 & 0.368 \\
\hline Smoking & $4(57.1 \%)$ & $15(68.2 \%)$ & 0.286 & 0.593 \\
\hline Drinking & $5(71.4 \%)$ & $14(63.6 \%)$ & 0.143 & 0.706 \\
\hline
\end{tabular}

divided into a TC group (22 patients) and a glossitis group (7 patients, including 1 atrophic glossitis, 2 median rhomboid glossitis, 1 geometric glossitis and 3 strawberry tongue) (Figures 1 and 2). There were no clinical or demographic differences between the two groups $(\mathrm{p}>0.05)$ (Table 1).

\section{Comparison of DCE-MRI Measurement Parameters Between the Two Groups}

The results of this study showed that the $K_{\text {trans }}$ value of the TC group was $0.96 \pm 0.34$, the $K_{\text {trans }}$ value of the glossitis group was $0.35 \pm 0.06$; the $K_{\text {trans }}$ value of the TC group was significantly higher than that of the glossitis group, and the difference was statistically significant $(P=0.000)$. The $K_{\text {ep }}$ value of the TC group was $1.55 \pm 0.46$, the $K_{\text {ep }}$ value of the glossitis group was $0.83 \pm 0.30$; the $K_{\text {ep }}$ value of the TC group was significantly higher than that of the glossitis group, and the difference was statistically significant $(P=0.001)$. The $\mathrm{CER}$ value of the TC group was 3.10 \pm 0.63 , the CER value of the glossitis group was $2.24 \pm$ 0.57 ; the CER value of the TC group was significantly higher than that of the glossitis group, and the difference was statistically significant $(P=0.004)$. The AUGG value of the patients in the TC group was $0.88 \pm 0.25$, and the AUGG value of patients in the glossitis group was $0.40 \pm$ 0.06; the AUGG value of patients in the TC group was significantly higher than that in the glossitis group, and the difference was statistically significant $(P=0.000)$. In addition, the values of $V_{\mathrm{e}}$, fPV, MaxSlope, and BAT measured by DCE-MRI in the TC group were higher than those in the glossitis group, but the difference was not statistically significant $(P>0.05)$. See Table 2 for details.
Table 2 Comparison of DCE-MRI Measurement Parameter Values Between Glossitis Group and Tongue Cancer Group $\overline{\mathrm{X}} \pm s$

\begin{tabular}{|l|l|l|l|l|}
\hline Parameter & $\begin{array}{l}\text { Glossitis } \\
\text { Group } \\
(\mathbf{n}=7)\end{array}$ & $\begin{array}{l}\text { Tongue } \\
\text { Cancer } \\
\text { Group } \\
(\mathbf{n}=\mathbf{2 2})\end{array}$ & T Value & $\boldsymbol{P}$ value \\
\hline$K_{\text {trans }} *$ & $0.35 \pm 0.06$ & $0.96 \pm 0.34$ & -7.761 & 0.000 \\
$V_{\mathrm{e}}$ & $0.51 \pm 0.18$ & $0.64 \pm 0.19$ & -1.763 & 0.108 \\
$K_{\text {ep }} *$ & $0.83 \pm 0.30$ & $1.55 \pm 0.46$ & -3.911 & 0.001 \\
fPV & $0.06 \pm 0.05$ & $0.13 \pm 0.18$ & -0.990 & 0.331 \\
MaxSlope & $0.083 \pm 0.063$ & $0.129 \pm 0.07$ & -1.647 & 0.111 \\
CER* & $2.24 \pm 0.57$ & $3.10 \pm 0.63$ & -3.181 & 0.004 \\
IAUGG* & $0.40 \pm 0.06$ & $0.88 \pm 0.25$ & -8.256 & 0.000 \\
BAT & $36.86 \pm 15.54$ & $37.61 \pm 43.05$ & -0.043 & 0.966 \\
\hline
\end{tabular}

Note: *Indicates significant difference between the two groups.

The Diagnostic Efficacy of Different Measured Parameter Values of DCE-MRI in Distinguishing Benign and Malignant

\section{Lesions}

The results of this study showed that the sensitivity of the $K_{\text {trans }}$ value $(0.484)$ measured by DCE-MRI to distinguish tongue inflammation from tumour was 0.995 , the specificity was 1.000 , the positive predictive value was 1.000 , and the negative predictive value was 0.857 . The sensitivity of the $K_{\text {ep }}$ value (1.151) for distinguishing tongue inflammation from tumour was 0.909 , the specificity was 0.857 , the positive predictive value was 0.950 , and the negative predictive value was 0.667 . The sensitivity of the IAUGG value (0.522) for distinguishing tongue inflammation from tumour was 0.909 , the specificity was 1.000 , the positive predictive value was 1.000 , and the negative predictive value was 0.778 . Therefore, the results of this study showed that the $K_{\text {trans }}$ value, the $K_{\text {ep }}$ value, and the IAUGG value measured by DCE-MRI had good ability to distinguish tongue inflammation from tumour and $\mathrm{K}_{\text {trans }}$ threshold of 0.484 has the best discriminative ability among them. The box plot of the four variable parameters, $K_{\text {trans }}, K_{\text {ep }}$, CER, and IAUGG, in the glossitis group and the TC group is shown in Figure 3. The ROC curves of the four variable parameters, $K_{\text {trans }}, K_{\text {ep }}$, CER, and IAUGG, to distinguish glossitis and TC are shown in Figure 4.

The sensitivity of CER to distinguish inflammation from tumour was 0.636 , the specificity was 1.000 , the positive predictive value was 1.000 , and the negative predictive value was 0.438 . Although there were significant 

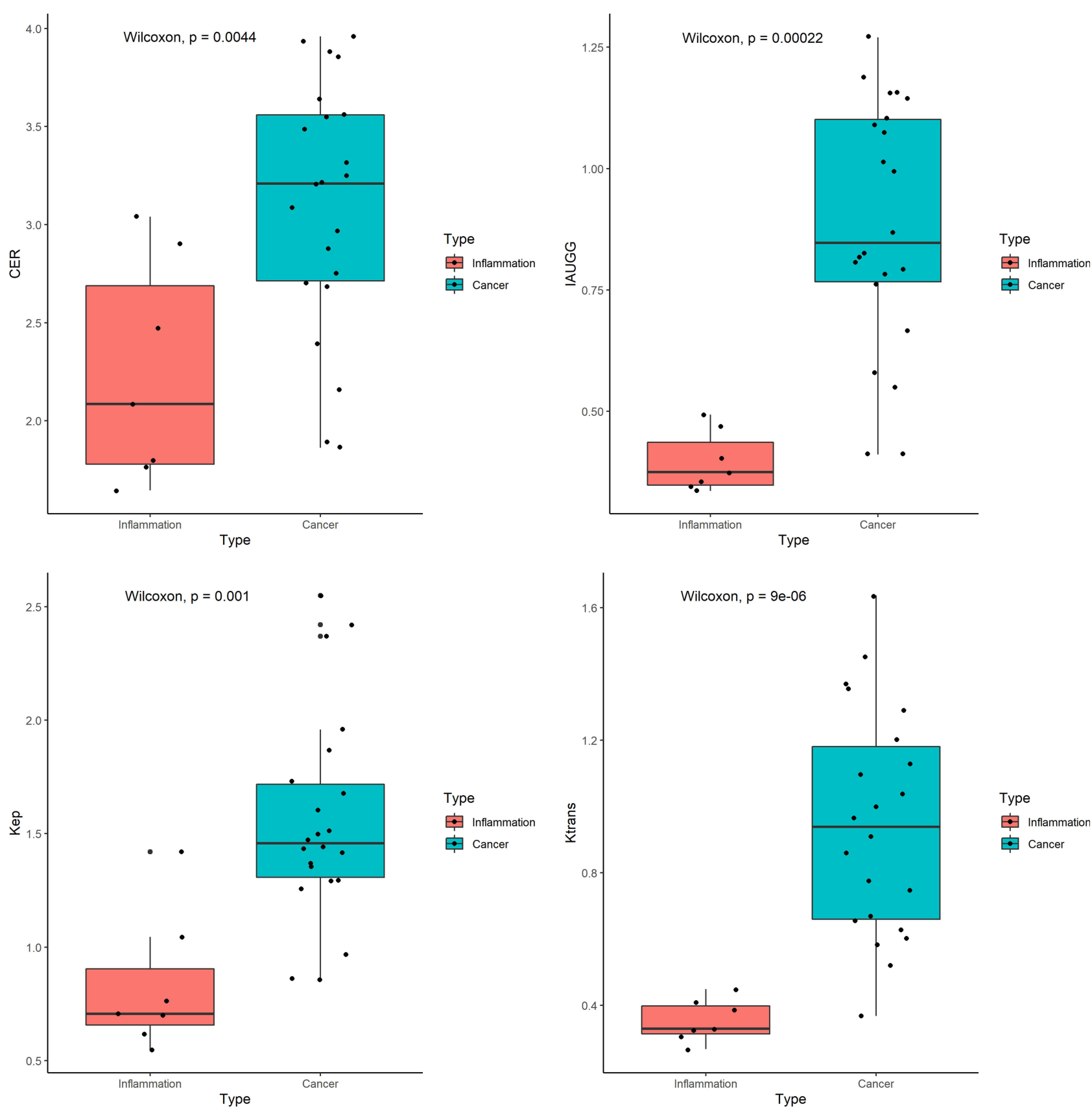

Figure 3 The "box plot" of the four variable parameters $K_{\text {trans }}, K_{\text {ep }}, C E R$, and IAUGG in the glossitis group and tongue cancer group.

differences in the CER parameter values between the two groups of samples, the values of the two groups were close, and the cut-off value almost overlapped with the mean value of the TC group. Simultaneously, the $95 \%$ confidence interval of its AUC was too large and was close to the threshold, and the sensitivity and negative predictive values were also low. Therefore, the ability of the CER to distinguish inflammation from tumour was poor. See Table 3 for details.

\section{Different Measured Parameter Values of DCE-MRI in Distinguishing the TNM Staging of TC}

The TC group $(n=22)$ was divided into the early stage group (stage I-II, $\mathrm{n}=10$ ) and the advanced stage group (stage III-IV, $\mathrm{n}=12$ ). The averages of the DCE-MRI parameters and their comparison between stage I-II and stage III-IV lesions were shown in Table 4. The mean $\mathrm{K}^{\text {trans }}$ values of stage I-II lesions were significantly higher than that of stage III-IV lesion $(\mathrm{p}=$ 

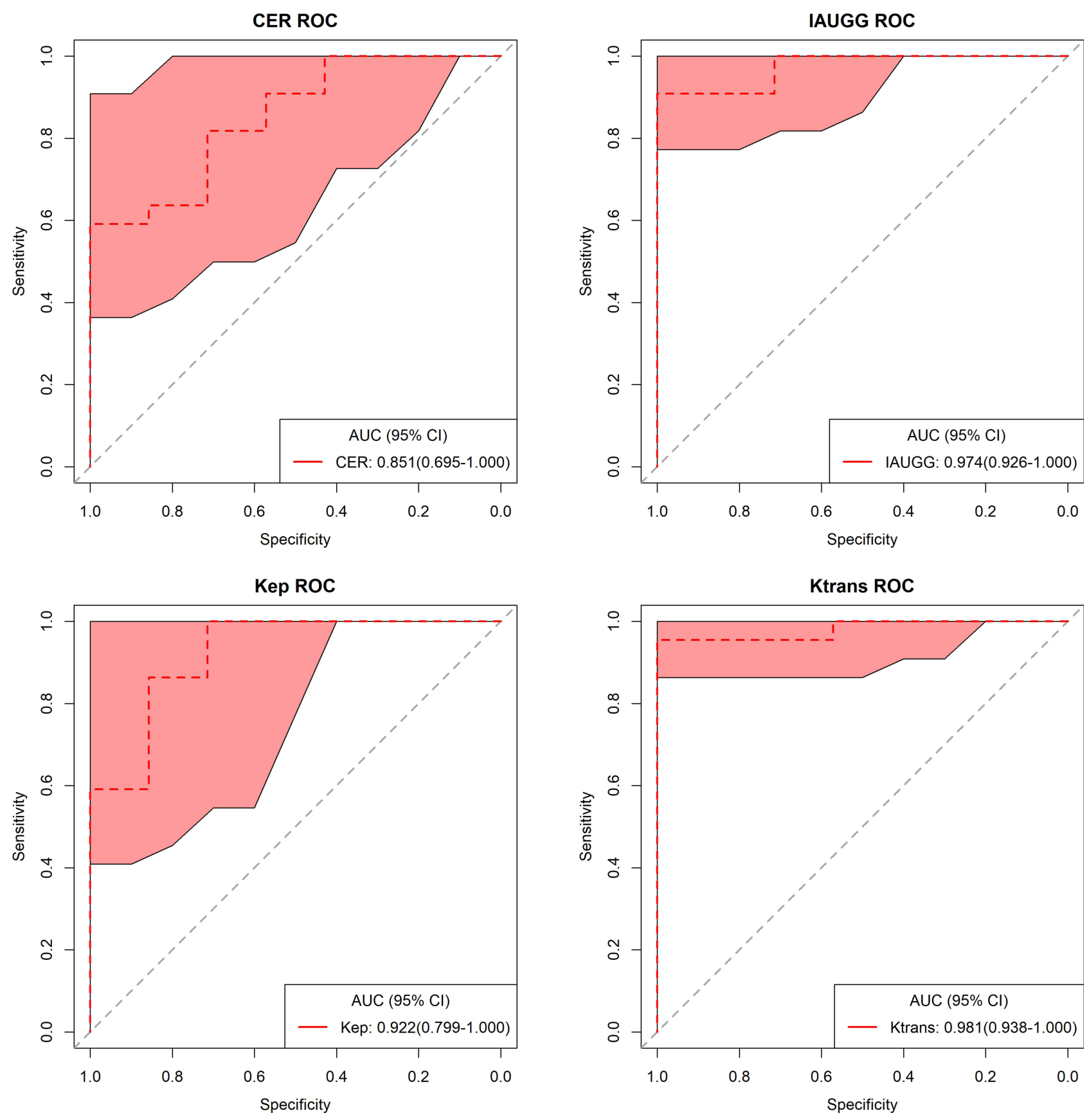

Figure 4 ROC curves of four variable parameters of $K_{\text {trans }}, K_{\text {ep }}$, CER, and IAUGG to distinguish glossitis from tongue cancer.

0.045), while there was no significant difference in other values $(\mathrm{p}>0.05)$. The AUC of $\mathrm{K}^{\text {trans }}$ was $73.3 \%$, and the sensitivity of the $\mathrm{K}^{\text {trans }}$ value for distinguishing the TNM staging of TC was 1.000 , the specificity was 0.917 , the positive predictive value was 0.644 , and the negative predictive value was 0.903 .

\section{Discussion}

The internal heterogeneity of the tumour and its microenvironmental characteristics reflect the biological behaviour, malignancy, responsiveness to treatment measures, and prognosis of the tumour. Because of its great advantages in soft tissue resolution and multiparameter imaging, MRI can be used as an effective inspection method to assess the internal heterogeneity and microenvironmental characteristics of tumours. Thus, it plays an important role in tumour diagnosis and differential diagnosis, grading and staging, treatment plan selection, and efficacy evaluation. 
Table 3 The Diagnostic Efficacy of Different Measured Parameter Values of DCE-MRI in Distinguishing Benign and Malignant Lesions $(95 \% \mathrm{Cl})$

\begin{tabular}{|l|l|l|l|l|l|l|}
\hline $\begin{array}{l}\text { Area Under } \\
\text { Variable Curve }\end{array}$ & $\begin{array}{l}\text { Susceptibility } \\
\text { (AUC) }\end{array}$ & Specificity (\%) & Specificity (\%) & $\begin{array}{l}\text { Positive } \\
\text { Predictive Value } \\
\text { (PPV) }\end{array}$ & $\begin{array}{l}\text { Negative } \\
\text { Predictive Value } \\
\text { (NPV) }\end{array}$ & $\begin{array}{l}\text { Cut- } \\
\text { Off } \\
\text { Vaule }\end{array}$ \\
\hline$K_{\text {trans }}$ & $0.981(0.938-1.000)$ & $0.955(0.864-1.000)$ & $1.000(0.286-1.000)$ & $1.000(1.000-1.000)$ & $0.875(0.667-0.875)$ & 0.484 \\
$K_{\text {ep }}$ & $0.922(0.799-1.000)$ & $0.909(0.455-1.000)$ & $0.857(0.429-1.000)$ & $0.950(0.909-0.957)$ & $0.667(0.500-0.700)$ \\
CER & $0.851(0.695-1.000)$ & $0.636(0.409-0.909)$ & $1.000(0.429-1.000)$ & $1.000(1.000-1.000)$ & $0.43(0.250-0.438)$ \\
IAUGG & $0.974(0.926-1.000)$ & $0.909(0.773-1.000)$ & $1.000(0.429-1.000)$ & $1.000(1.000-1.000)$ & $0.778(0.600-0.778)$ & 0.522 \\
\hline
\end{tabular}

Table 4 Different Parameter Values of DCE-MRI in Distinguishing the TNM Staging of TC $(95 \% \mathrm{Cl})$

\begin{tabular}{|l|l|l|l|l|}
\hline Parameter & $\begin{array}{l}\text { Stage I-II } \\
(\mathbf{n}=\mathbf{1 0})\end{array}$ & $\begin{array}{l}\text { Stage III-IV } \\
(\mathbf{n}=\mathbf{I})\end{array}$ & T Value & $\boldsymbol{P}$ value \\
\hline$K_{\text {trans }} *$ & $1.099 \pm 0.359$ & $0.821 \pm 0.278$ & 2.044 & 0.045 \\
$V_{\mathrm{e}}$ & $0.682 \pm 0.21 \mathrm{I}$ & $0.617 \pm 0.17 \mathrm{I}$ & 0.804 & 0.431 \\
$K_{\text {ep }}$ & $1.688 \pm 0.406$ & $1.443 \pm 0.483$ & 1.268 & 0.219 \\
fPV & $0.103 \pm 0.055$ & $0.155 \pm 0.053$ & -0.634 & 0.533 \\
MaxSlope & $0.114 \pm 0.046$ & $0.153 \pm 0.068$ & -1.554 & 0.136 \\
CER & $3.151 \pm 0.661$ & $3.059 \pm 0.638$ & -0.333 & 0.743 \\
IAUGG & $0.967 \pm 0.284$ & $0.816 \pm 0.216$ & 1.421 & 0.171 \\
BAT & $49.861 \pm 12.947$ & $27.502 \pm 7.141$ & 1.220 & 0.237 \\
\hline
\end{tabular}

Note: *Indicates significant difference between the two groups.

Previous histopathological examination is the gold standard for evaluating tumour heterogeneity, but the information about tumour tissue and microenvironment obtained by puncture biopsy or surgery has great limitations, and cannot reveal the overall situation of the tumour tissue, as well as having poor repeatability, certain traumas and risks, and coming from an invasive examination. Imaging analysis can quantitatively analyse tumour heterogeneity through specific parameters under non-invasive conditions; then, the overall situation of living tumours can be obtained with high repeatability. Imaging analysis has important clinical significance for studying the occurrence and development of tumours and for formulating more precise individualised medical treatment plans.

Traditional imaging techniques (including $\mathrm{CT}$ and conventional static MRI) usually use descriptive terms including lesion shape, size, surrounding invasion, and internal structure. Among these terms, size is the most commonly used diagnostic criterion, and internal necrosis is regarded as the most reliable sign of malignancy. However, some reports suggested that tumor margin, homogeneity, and signal intensity were not the differentiating factors for the correct differentiation between benign and malignant diseases. ${ }^{14,15}$ Malignant tumors were characterized by deep structural infiltration, while inflammatory diseases and malignancies were characterized by subcutaneous fatty infiltration. ${ }^{16}$ Therefore, in the process of clinical application, these signs alone are not sufficient to completely distinguish benign and malignant tumours. These subjective analysis models based on conventional parameters cannot quantify tumour heterogeneity, nor can they reflect the changes in the tumour's internal and surrounding microenvironment; they can no longer meet the current clinical needs of tumour precision diagnosis and treatment evaluation.

DCE-MRI is an imaging technique that reflects the blood perfusion status of the tissue's microcirculation. ${ }^{21,22}$ Commonly used semiquantitative parameters are: time-to-intensity curve, maximum slope and peak time, etc. Commonly used quantitative parameters include: volume transfer constant (Ktrans), rate constant (Kep), extravascular extracellular volume fraction (Ve), etc. he results of this study showed that the Ktrans, Kep, CER, and AUGG values of the TC group were significantly higher than those of the glossitis group, and the difference was statistically significant. Chen et al reported that the values of both Ktrans and ve of normal tissue differed significantly from those of nodes and primary tumours respectively. ${ }^{23}$ In addition, in terms of the diagnostic efficiency of differentiating benign and malignant lesions, Ai et al reported that the combination of DCE-MRI and DW-MRI improved the diagnostic accuracy in differentiating benign lesions from malignant tongue tumours. ${ }^{21}$ The results of this study showed that the Ktrans, Kep, and IAUGG values measured by DCE-MRI had good ability to distinguish tongue inflammation from tumours and $\mathrm{K}_{\text {trans }}$ threshold of 0.484 has the best discriminative ability among them. In addition, the mean Ktrans 
values of stage I-II lesions were significantly higher than that of stage III-IV lesion, which was consistent with the study of Guo et al. ${ }^{19}$ Thus, DCE-MRI can effectively distinguish the benign and malignant tongue lesions, and the TNM staging of malignant tongue lesions, which lays a foundation for its clinical application.

This research retains the following shortcomings. First, this study is a case-control study, not a randomised controlled experiment. Second, this study is a single-centre clinical study, and the sample size included is relatively small. It remains necessary to increase the sample size and conduct multi-centre clinical research. Finally, the paper lacks any quantitative comparison between the new method and any previous diagnostic method. In the future work, it will be more meaningful to continuously include new cases and conduct a controlled study on the accuracy of the new and other techniques.

\section{Conclusion}

DCE-MRI is effective in distinguishing benign and malignant tongue lesions and the internal heterogeneity of the tumour; it is worth following up in a larger study.

\section{Data Sharing Statement}

The datasets used and/or analysed during the current study are available from the corresponding author on reasonable request.

\section{Ethics Approval}

This study was conducted in accordance with the Declaration of Helsinki and approved by the ethics committee of Cangzhou Central Teaching Hospital (IRB:2017-027).

\section{Consent for Publication}

All authors agree to publish this article.

\section{Informed Consent}

Informed consent has been obtained from all individuals included in this study.

\section{Acknowledgements}

The authors thank Jia-Liang Ren (GE Healthcare China) for providing help on the experimental equipment and instruments for valuable results.

\section{Disclosure}

The authors declare that they have no competing interests.

\section{References}

1. Gaelen SM, Bradshaw PT, Weissler MC, et al. Interaction between known risk factors for head and neck cancer and socioeconomic status: the Carolina Head and Neck Cancer Study. Cancer Causes Control. 2018;29(9):863-873. doi:10.1007/s10552-018-1062-8

2. Colevas AD, Yom SS, Pfister DG, et al. NCCN guidelines insights: head and neck cancers, version 1. JNCCN. 2018;16 (5):479-490.

3. Francis D. Trends in incidence of head and neck cancers in India. Eur $J$ Cancer. 2018;92:S23. doi:10.1016/j.ejca.2018.01.056

4. Schantz SP, Yu GP. Head and neck cancer incidence trends in young Americans, 1973-1997, with a special analysis for tongue cancer. Arch Otolaryngol Head Neck Surg. 2002;128(3):268. doi:10.1001/ archotol.128.3.268

5. Polednak AP, Phillips C. Cancers coded as tongue not otherwise specified: relevance to surveillance of human papillomavirus-related cancers. J Registry Manag. 2014;41(4):190-195.

6. Attner P, Du J, Näsman A, et al. Human papillomavirus and survival in patients with base of tongue cancer. Int J Cancer. 2011;128 (12):2892-2897. doi:10.1002/ijc. 25625

7. Tertipis N, Villabona L, Nordfors C, et al. HLA-A*02 in relation to outcome in human papillomavirus positive tonsillar and base of tongue cancer. Anticancer Res. 2014;34(5):2369-2375.

8. Attner P, Du J, Näsman A, et al. The role of human papillomavirus in the increased incidence of base of tongue cancer. Int $J$ Cancer. 2010;126(12):2879-2884.

9. Liang XH, Lewis J, Foote R, et al. Prevalence and significance of human papillomavirus in oral tongue cancer: the Mayo Clinic experience. J Oral Maxillofacial Surg. 2008;66(9):1875-1880. doi:10.1016/j.joms.2008.04.009

10. Kabeya M, Furuta R, Kawabata K, et al. Prevalence of human papillomavirus in mobile tongue cancer with particular reference to young patients. Cancer Sci. 2012;103(2):161-168.

11. Domino FJ, Baldor RA, editors. The 5-Minute Clinical Consult 2012. 20th ed. Philadelphia, Pa: Wolters Kluwer Health/Lippincott Williams \& Wilkins; 2012:532-533. ISBN 978-1451103038.

12. Sharabi AF, Winters R. Glossitis. In: StatPearls [Internet]. Treasure Island (FL): StatPearls; 2021. Available from https://www.ncbi.nlm. nih.gov/books/NBK560627/.

13. Byrd JA, Bruce AJ, Rogers RS. Glossitis and other tongue disorders. Dermatol Clin. 2003;21(1):123-134. doi:10.1016/S0733-8635(02) 00057-8

14. Freling NJ, Molenaar WM, Vermey A, et al. Malignant parotid tumors: clinical use of MR imaging and histologic correlation. Radiology. 1992;185:691-696. doi:10.1148/radiology.185.3.1438746

15. Teresi LM, Lufkin RB, Wortham DG, et al. Parotid masses: MR imaging. Radiology. 1987;163:405-409. doi:10.1148/ radiology.163.2.3562818

16. Swartz JD, Rothman MI, Marlowe FI, et al. MR imaging of parotid mass lesions: attempts at histopathologic differentiation. J Comput Assist Tomogr. 1989;13:789-796. doi:10.1097/00004728-19890900000007

17. Quon H, Kim S, Loevner L, et al. DCE-MRI of metastatic nodal head and neck squamous cell carcinomas: feasibility and preliminary evidence of a relationship between perfusion and pathologic residual neck disease. Int J Radiat Oncol Biol Phys. 2007;69(3):425-426. doi:10.1016/j.ijrobp.2007.07.1575

18. Zheng N, Li R, Liu W, et al. The diagnostic value of combining conventional, diffusion-weighted imaging and dynamic contrast-enhanced MRI for salivary gland tumors. $\mathrm{Br} J$ Radiol. 2018;91(1089):20170707. doi:10.1259/bjr.20170707

19. Guo N, Zeng W, Deng H, et al. Quantitative dynamic contrast-enhanced MR imaging can be used to predict the pathologic stages of oral tongue squamous cell carcinoma. BMC Med Imaging. 2020;20:117. doi:10.1186/s12880-020-00516-w 
20. Ridge JA, Lydiatt WM, Patel SG, et al. Oral cavity. In: Amin MB, editor. AJCC Cancer Staging Manual. Vol. II. 8th ed. Chicago: American College of Surgeons; 2017:79-94.

21. Ai ST, Zhu WJ, Liu Y, et al. Combined DCE- and DW-MRI in diagnosis of benign and malignant tumors of the tongue. Front Biosci. 2013;18(18):1098-1111.

22. Duan C, Kallehauge JF, Bretthorst GL, et al. Are complex DCE-MRI models supported by clinical data? Magn Resonan Med. 2017;77 (3):1329. doi:10.1002/mrm.26189
23. Chen L, Ye Y, Chen H, et al. Dynamic contrast-enhanced magnetic resonance imaging for differentiating between primary tumor, metastatic node and normal tissue in head and neck cancer. Curr Med Imaging Rev. 2018;14(3):416-421. doi:10.2174/157340561466617 1205105236

\section{Publish your work in this journal}

Cancer Management and Research is an international, peer-reviewed open access journal focusing on cancer research and the optimal use of preventative and integrated treatment interventions to achieve improved outcomes, enhanced survival and quality of life for the cancer patient.
The manuscript management system is completely online and includes a very quick and fair peer-review system, which is all easy to use. Visit http://www.dovepress.com/testimonials.php to read real quotes from published authors. 